\title{
Towards traps for cold molecules
}

A scheme for trapping molecules in strong infrared laser beams has been described theoretically by two chemists. Experimental proof awaits. When it comes, molecular spectroscopy will be transformed.

CoOling atoms to low temperatures is now almost child's play. The simplest thing to do is to start with ions, decelerate them in an electrostatic field and then catch them in an electromagnetic trap which may be either a geometrical arrangement of electrostatic and magnetic fields or simply a pattern of electromagnetic forces as generated by mutually perpendicular Helmholtz coils on a very small scale. Then it is a matter of robbing the ions of kinetic energy, for which purpose laser light detuned from a resonance line seems to be the most effective means. Temperatures of a millikelvin are accessible. The benefits to spectroscopy are immense.

Molecules, sadly, are much less easily trapped and cooled. Ionization is often a prelude to dissociation, the electrons in an ionized molecule may otherwise be redistributed into different states and there is the complexity of rotation and vibration as well as excitation to contend with. Even so, there are some experiments that suggest that molecules may be aligned with each other by high-intensity laser pulses provided that they are sufficiently brief to avoid dissociation - a picosecond or so.

That seems to be the starting point for a remarkable article by two Harvard chemists, Bretislav Friedrich and Dudley Herschbach, which essentially outlines a scheme for trapping and cooling molecules of most simple kinds (Phys. Rev. Lett. 74, 4623-4626; 1995). The remarkable feature of this study is that it is entirely a theoretical study.

In molecular cooling, the alignment of molecules is the first need. If a molecule has a dipole moment, simple electrostatic fields should do the trick. Of course, the accuracy of the alignment will turn on how much kinetic energy an ensemble of molecules has available for oscillation about the dipole axis. One technique for doing this is to cool the molecules (enclosed in a good vacuum) by the vapour from a pool of liquid ${ }^{3} \mathrm{He}$, which can rob an ensemble of dipolar molecules of their rotational kinetic energy.

But what if the molecules carry no permanent dipole moment? That is the point from which Friedrich and Herschbach take off. Whether or not molecules have permanent dipoles, they are polarizable in electric fields. What better, to induce that condition, than an intense laser beam? But how do molecules behave in such conditions? On the face of things, it is simply a matter of solving a Schrödinger equa- tion for the angular rotation of a molecule dealt with as if it were a rigid rotator vibrations are, in other words, neglected.

The problem is tractable if the frequency of the radiation is greater than the reciprocal of the duration of the laser pulse. Then the effects of whatever permanent dipole moment there may be are averaged out. The results of the calculation are interesting only if the polarizability of the molecule is asymmetric, which is usually the case. For a diatomic molecule, for example, the polarizability along the internuclear axis and in the perpendicular direction will almost certainly be different. But if the polarizability in two directions should be equal, the rotation of the molecule would correspond to the rotation of a molecule in the absence of an electric field, which provides a neat way of classifying the states of the molecule in the presence of a field in terms of the angular momentum of the molecule and its projection along the axis.

The eventual outcome is a calculation of the statistical properties of a collection of molecules in the intense electric field of a powerful laser beam in the infrared region of the spectrum. Evidently the degree of alignment of molecules at any temperature will increase with the rotational constant (or moment of inertia) of the rotating molecule. The greater the temperature, the less good the alignment will be. But for anisotropic molecules and at lower temperatures, the mean-square of $\cos \theta$, where $\theta$ is the angle between the orientation of the molecule and its axis of polarizability, is arbitrarily close to unity; alignment should be straightforward.

In due course, these calculations will find their way into the textbooks of quantum mechanics. The states of a molecule in an electric field strong enough for the polarization to be significant are called "pendular" states. They should provide spectroscopists with a field day; the selection rules determining which states can be converted into others by emission or absorption of infrared radiation are shown to be different from those familiar to molecular spectroscopists.

How likely is it that these conditions can be achieved in a real laboratory? Friedrich and Herschbach applaud the neatness of the experiments at Saclay in which D. Normand, L. A. Lompre and C. Cornaggia first demonstracted alignment in CO molecules (J. Phys. B 25, L497; 1992), in which liquid ${ }^{3} \mathrm{He}$ was first used for cooling. The difficulty will be that of making laser beams intense enough not merely to win good alignment of molecules but to trap them in very small places.

That should work because polarizable molecules should gravitate to regions in which the intensity of the field is greatest. So much is true in classical physics, but the quantum mechanical calculations bear out the expectation. It should then suffice as a trap for molecules to arrange that the laser should concentrate its energy into a volume of $10^{-9} \mathrm{~cm}^{3}$ or $10 \mu \mathrm{m}$ in size. They reckon that it would not be necessary to use a pulsed laser, and that a continuouswave laser delivering a few kilowatts of power would suffice if its energy were concentrated in the trapping volume by sufficiently accurate mirrors.

By the same test, the use of liquid ${ }^{3} \mathrm{He}$ for robbing the gas molecules of translational and vibrational energy should suffice. The technique is to keep the liquid helium in a cryostat and to allow its natural vapour pressure to do the cooling. Obviously it is necessary that the vapour pressure should be high enough to carry away the excess energy. According to Friedrich and Herschbach, the energy states of the trapped molecules should be lower than the energy of escape by the equivalent of $1 \mathrm{~K}$.

They are even able to make predictions of the trapping process for three molecules - $\mathrm{CO}, \mathrm{CS}_{2}$ and $\mathrm{C}$. They reckon that they should be able to achieve concentrations between $10^{8}$ and $10^{13} \mathrm{~cm}^{-3}$ with suitable lasers and coolant gas. These are not very different from the concentrations the atom trappers boast about.

There is even the possibility of further cooling of an ensemble of trapped molecules by allowing those with the highest energy to escape periodically. 'Evaporative' cooling on this model is used in cooling trapped atoms, and should work just as well for atoms.

So why have Friedrich and Herschbach not carried out the measurements whose outcome they predict with such exquisite certainty? There are some obvious reasons why they should hang back; theoreticians are not always natural experimentalists. But then it is an act of great generosity on their part to have given some group somewhere a detailed protocol that could almost certainly be a successful grant application overnight.

John Maddox 